TRANSACTIONS OF THE

AMERICAN MATHEMATICAL SOCIETY

Volume 313, Number 2, June 1989

\title{
ON THE HOPF INDEX AND THE CONLEY INDEX
}

\author{
CHRISTOPHER K. MCCORD
}

\begin{abstract}
The following generalization of the Poincare-Hopf index theorem is proved: If $S$ is an isolated invariant set of a flow on a manifold $M$, then the sum of the Hopf indices on $S$ is equal (up to a sign) to the Euler characteristic of the homology Conley index of $S$.
\end{abstract}

One of the classical results in dynamical systems relating local and global information is the Poincare-Hopf index theorem. If $\mathscr{Z}$ is a vectorfield on a compact manifold $M$ with isolated zeros, then the intersection number of $\mathscr{X}$ with the zero section of $T M$ is defined to be the sum of the Hopf indices of the zeros: $I(\mathscr{X}) \equiv \sum l_{x}(\mathscr{X})$. If $M$ is without boundary or if $\mathscr{X}$ is outward on $\partial M$, the Poincaré-Hopf theorem asserts that the intersection number of $\mathscr{Z}$ is equal to the homological Euler characteristic of $M: \chi(M)=I(\mathscr{X})$. See $[4,7]$.

This has the restriction that the local information (the Hopf indices) is only related to the global information (the Betti numbers) when the vectorfield is outward on the boundary. Pugh [8] removes this restriction in the case of generic contact of $\mathscr{Z}$ with $\partial M$ by relating the intersection number to a sum of Euler characteristics: $I(\mathscr{Z})=\chi(M, \partial M)+\sum \chi\left(R_{-}^{i}, \Gamma^{i}\right)$, where $R_{-}^{i}$ is the " $i$-codimensional exit region" and $\Gamma^{i}$ is its boundary.

In this paper, we further develop this result by relating the Hopf index and intersection numbers to the Conley index for dynamical systems. Suppose that $\chi$ is a smooth vectorfield on $M$, with $S \subseteq M$ an isolated invariant set of the induced flow. Then the intersection number of $\mathscr{X}$ on a neighborhood of $S$ is (up to a sign) the Euler characteristic of the homology Conley index of $S$ in $M$, the Conley-Euler number $\chi(M ; S)$. In particular, if all zeros on $\mathscr{Z}$ on $S$ are nondegenerate, then the Conley index and the Hopf index are related by the formula $(-1)^{n} \chi(M ; S)=\sum l_{x}(\mathscr{X})$.

There are two aspects to this result. First, it gives the Hopf index and intersection numbers a homological interpretation in a broad setting. Second, it shows that the properties of the Hopf index, such as additivity, stability, etc., are also properties of Conley-Euler numbers. To develop the theorem, we first

Received by the editors February 8, 1988.

1980 Mathematics Subject Classification (1985 Revision). Primary 34C35, 55M20; Secondary $54 \mathrm{H} 20,58 \mathrm{~F} 25$.

Work supported in part by OARPA Applied and Computational Mathematics Program. 
describe the relevant portions of the Conley index theory (cf. $[2,10])$ and establish the appropriate notation for comparing the Hopf and Conley indices.

Given a flow on a manifold $M$, a set $S \subseteq M$ is an isolated invariant set if there exists a compact set $N$ with $S \subset$ int $_{M}(N)$ and the maximal invariant set in $N$. Given such an $S$, a compact pair $\left(N, L^{+}\right)$is an index pair for $S$ in $M$ if $\operatorname{cl}\left(N \backslash L^{+}\right)$is an isolating neighborhood for $S$ and $L^{+}$is a positively invariant exit set for $N$ (i.e. any orbit exiting $N$ in forward time first passes through $\left.L^{+}\right)$. A similar construction may be made in the reverse flow, producing an index pair $\left(N, L^{-}\right)$, with $L^{-}$a negatively invariant entrance set for $N$. Such index pairs exist for all $S$, and the homotopy type of $N / L^{+}$is independent of the index pair chosen. This homotopy type defines the Conley index of $S$ in $M$.

Robbin and Salamon [9] present a construction of index pairs which will be of particular use to us. By construction a smooth Lyapunov function on a neighborhood of $S$, they show that a triple $\left(N, L^{-}, L^{+}\right)$can be chosen with $N$ an isolating neighborhood for $S$ with $\partial N=L^{-} \cup L^{+}$, and with the flow entering $N$ along $L^{-}$and existing along $L^{+}$. Further, $L^{+}$and $L^{-}$can be chosen to be $(n-1)$-manifolds with boundary, so that $N$ is a "manifold with corners," with those corners contained in $L^{-} \cap L^{+}$. Then $\left(N, L^{+}\right)$is an index pair for $S$ in the forward flow; and $\left(N, L^{-}\right)$is an index pair for $S$ in the reverse flow. We will refer to such a triple $\left(N, L^{-}, L^{+}\right)$as an index triple for $S$ in $M$.

The homology Conley index of $(M ; S)$ is defined by

$$
C H_{*}^{+}(M ; S)=H_{*}\left(N, L^{+}\right),
$$

and the Conley-Euler characteristic of $(M ; S), \chi^{+}(M ; S)$, is defined to be the Euler characteristic of $C H_{*}^{+}(M ; S)$. We will assume that $S \cap \partial M=\varnothing$. If $M$ is orientable, the homology indices in the forward and reverse flows are related by a duality isomorphism $D_{*}: C H_{-}^{*}(M ; S) \rightarrow C H_{*}^{+}(M ; S)$ [6], and the ConleyEuler characteristics are equal up to a sign: $\chi^{+}(M ; S)=(-1)^{n} \chi^{-}(M ; S)$. If only the forward index is involved, we will write $\mathrm{CH}_{*}(M ; S)$ for $\mathrm{CH}_{*}^{+}(M ; S)$ and $\chi(M ; S)$ for $\chi^{+}(M ; S)$.

Stability in the Conley index is described by continuation. Let $\Lambda$ be a pathconnected parameter space and consider a family of vectorfields $\mathscr{X}_{\lambda}$ on $M$ parametrized by $\Lambda$. Let $M_{\lambda}$ denote $M$ with the flow generated by $\mathscr{X}_{\lambda}$. Form a space $\mathscr{S}(\Lambda)=\left\{\left(S_{\lambda}, \lambda\right) \subseteq M \times \Lambda: S_{\lambda}\right.$ is an isolated invariant set in $\left.M_{\lambda}\right\}$. For every compact $N \subseteq M$, let $\Lambda(N)$ be the set of parameter values for which $N$ is an isolating neighborhood. $\Lambda(N)$ is open in $\Lambda$. There is then a function $\sigma_{N}: \Lambda(N) \rightarrow \mathscr{S}(\Lambda)$ defined by $\sigma_{N}(\lambda)=\left(S_{\lambda}, \lambda\right)$, where $S_{\lambda}$ is the maximal invariant set of $N$ in $M_{\lambda}$. Topologize $\mathscr{S}(\Lambda)$ by taking as basic sets $\sigma_{N}(U)$, where $U$ is open in $\Lambda(N)$. In this topology, $\mathscr{S}(\Lambda)$ is not Hausdorff, but the natural projection is a local homeomorphism. Two isolated invariant sets are 
said to be related by continuation if they lie in the same path component of $\mathscr{S}(\Lambda)$.

The Conley index is stable under continuation. Indeed, Robbin and Salamon show that, in the smooth setting, index triples are stable on open sets in $\mathscr{S}(\Lambda)$ : if $\left(N, L^{-}, L^{+}\right)$is an index triple for the maximal invariant set of $N$ at one parameter value, it remains an index triple for the maximal invariant set in $N$ at nearby parameter values. The Conley-Euler number then defines an integer-valued function on $\mathscr{S}(\Lambda), \chi: \mathscr{S}(\Lambda) \rightarrow \mathbf{Z}$, which is constant on path components.

It will be useful to reformulate the Hopf index in this language. An isolated critical point set of a vectorfield is a set of critical points $C$ with a compact neighborhood $N$ such that $C \subset$ int $_{M} N$ and $C$ is the maximal critical point set in $N$ (i.e. there are no critical points in $N \backslash C$ ). Given a parameterized family of vectorfields $\mathscr{X}_{\lambda}$, let $\mathscr{C}(\Lambda)=\left\{\left(C_{\lambda}, \lambda\right): C_{\lambda}\right.$ is an isolated critical point set for $\left.\mathscr{X}_{\lambda}\right\}$. For every compact $N \subseteq M$, let $\Lambda(N)$ be the (open) set of parameter values for which $\mathscr{Z}_{\lambda}$ is nonzero on $\partial N$. Define $\kappa_{N}: \Lambda(N) \rightarrow \mathscr{C}(\Lambda)$ by $\kappa_{N}(\lambda)=\left(C_{\lambda}, \lambda\right)$, where $C_{\lambda}$ is the zero set of $\mathscr{\mathscr { X }}_{\lambda}$ on $N$. Topologize $\mathscr{C}(\Lambda)$ by taking as basic sets $\kappa_{N}(U)$, where $U$ is open in $\Lambda(N)$. Two critical point sets are related by continuation if they lie in the same path component of $\mathscr{C}(\Lambda)$.

For every element of $\mathscr{C}(\Lambda)$, there is an isolating neighborhood $N$ which is a manifold with boundary. The intersection number of $\mathscr{Z}_{\lambda}$ with the zero section $Z(M)$ of the tangent bundle then defines a function $I: \mathscr{C}(\Lambda) \rightarrow \mathbf{Z}$, which is constant on path-components of $\mathscr{C}(\Lambda)$. Recall that a critical point $x$ is nondegenerate if $d \mathscr{X}_{x}: T_{x} M \rightarrow T_{x} M$ is nonsingular. The fixed-point index of $\mathscr{X}$ at $x, l_{x}(\mathscr{X})$, is given by the sign of the determinant of $d \mathscr{X}_{x}$. Let $\mathscr{C}^{\prime}(\Lambda) \subseteq \mathscr{C}(\Lambda)$ consist of the critical point sets with all critical points nondegenerate. Then on $\mathscr{C}^{\prime}(\Lambda), I$ is equal to the sum of the fixed-point indices: $I\left(C_{\lambda}, \lambda\right)=\sum_{x \in C_{\lambda}{ }^{l}}\left(\mathscr{X}_{\lambda}\right)$.

If $\left(S_{\lambda}, \lambda\right) \in \mathscr{S}(\Lambda)$ with isolating neighborhood $N$, then there are no zeros of $\mathscr{X}_{\lambda}$ on $\partial N$, and $\kappa_{N}(\lambda)=\left(C_{\lambda}, \lambda\right)$ is an isolated critical point set. That is, there is a map $R: \mathscr{S}(\Lambda) \rightarrow \mathscr{C}(\Lambda)$ defined by $R\left(S_{\lambda}, \lambda\right)=\left(C_{\lambda}, \lambda\right)$, with $R \circ \sigma_{N}=\kappa_{N}$. Note that $R$ may not be one-to-one, nor onto. There can be several isolated invariant sets with the same critical point set, and there can be critical points which are not contained in any isolated invariant set. Our main result shows that $R$ relates the Hopf index and the Conley-Euler number.

Theorem. Given a family of $C^{\infty}$ vectorfields on $M$ parameterized by $\Lambda$, there is a commutative diagram

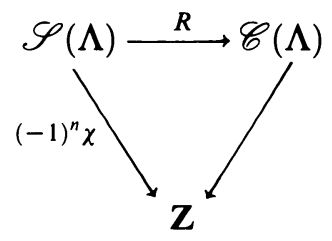




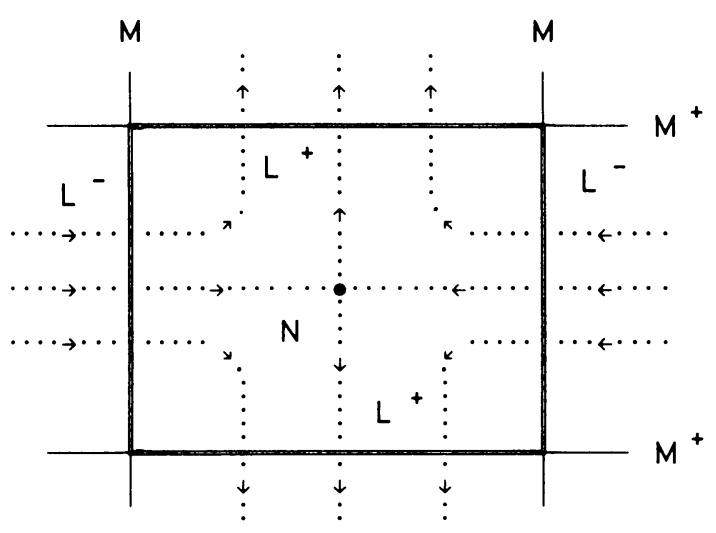

(a)

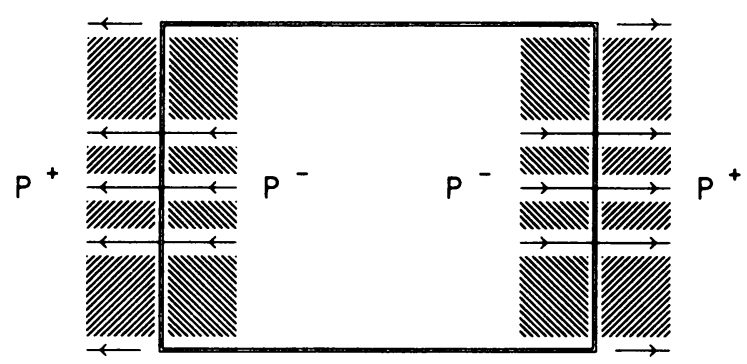

(b)

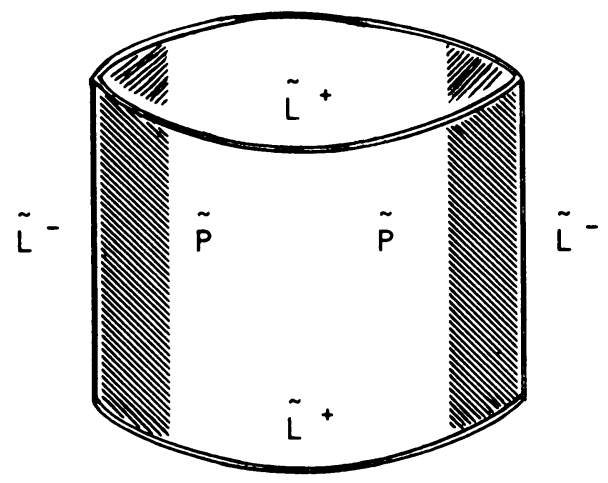

(c)

\section{Figure 1}

That is, the Conley-Euler number of $S_{\lambda}$ (up to a sign) is the intersection number of $\mathscr{X}_{\lambda}$ on its critical point set. In particular, if all critical points of $\mathscr{X}_{\lambda}$ on $S_{\lambda}$ are nondegenerate, then $(-1)^{n} \chi\left(M_{\lambda} ; S_{\lambda}\right)=\sum_{R\left(S_{\lambda}\right)} l_{x}\left(\mathscr{X}_{\lambda}\right)$. 
Proof. Fix $\left(S_{\lambda}, \lambda\right) \in \mathscr{S}(\Lambda)$. If $R\left(S_{\lambda}, \lambda\right) \notin \mathscr{C}^{\prime}(\Lambda)$, an arbitrarily small perturbation $\mathscr{X}_{\mu}$ of $\mathscr{X}_{\lambda}$ (in some space of vectorfields $K$, but not necessarily in $\Lambda$ ) has $R\left(S_{\mu}, \mu\right) \in \mathscr{C}^{\prime}(K)$. As $\chi$ and $I$ are constant on path components of $\mathscr{S}(K)$, $\chi\left(M_{\lambda} ; S_{\lambda}\right)=\chi\left(M_{\mu} ; S_{\mu}\right)$ and $I\left(M_{\lambda} ; C_{\lambda}\right)=I\left(M_{\mu} ; C_{\mu}\right)$, so we may assume without loss that $R\left(S_{\lambda}, \lambda\right) \in \mathscr{C}^{\prime}(\Lambda)$. Choose an index triple $\left(N, L^{-}, L^{+}\right)$for $S$ in $M_{\lambda}$.

Form the double $\tilde{N}$ of $N$ along $L^{-}$as follows: $L^{ \pm}$is the intersection with $N$ of a $(n-1)$ submanifold $M^{ \pm}$, with $M^{+}$and $M^{-}$transverse (Figure 1a). Choose a tangent vectorfield on $M^{+}$which is transverse to $M^{-}$and tangent to $M^{+}$along $M^{+} \cap M^{-}$. This extends to a vectorfield $\mathscr{Y}$ on $M$ which is transverse to $M^{-}$on a neighborhood of $L^{+} \cap L^{-}$and tangent to $M^{+}$. The original vectorfield $\mathscr{Z}$ is transverse to $L^{-}$, so there exists a smooth vectorfield $\mathscr{P}(x)=f_{1}(x) \cdot \mathscr{X}(x)+f_{2}(x) \cdot \mathscr{Y}(x)$ which is transverse to $M^{-}$on $L^{-}$and tangent to $M^{+}$. Denote $\mathscr{P}$ solution curves by $\{x \circ t: t \in \mathbf{R}\}$. Assume that the vectorfield points outward from $N$ on $L^{-}$, and choose $\varepsilon>0$ so that the set of $\mathscr{P}$-solution curves $P=\left\{x \circ t: x \in L^{-},-\varepsilon<t<\varepsilon\right\}$ is a smooth embedding of $L^{-} \times(-\varepsilon, \varepsilon)$ and $P^{+}=\left\{x \circ t: x \in L^{-}, 0<t<\varepsilon\right\}$ does not intersect $N$ (Figure 1b). Then $P^{+}$collars $L^{-}$along $N$, and $N^{\prime}=N \cup P$ is a manifold with boundary. In $N^{\prime}, L^{-}$is a neat submanifold with $P$ a tubular neighborhood for it. Form the double $\tilde{N}$ by taking $N^{\prime} \times\{0,1\}$ and identifying $(x \circ t, 0)$ with $(x \circ(-t), 1)$ for $x \circ t \in P$ (Figure 1c). For any $U \subseteq N$, let $\widetilde{U}$ denote the image of $U \times\{0,1\}$ in $\tilde{N}$. Then $\tilde{N}$ is a smooth manifold with boundary $\widetilde{L}^{+}$, and $L^{-}$is a neat submanifold with tubular the neighborhood $\widetilde{P}^{-}$.

The goal is to define a vectorfield $\widetilde{\mathscr{X}}$ on $\tilde{N}$ which agrees with $\mathscr{Z}$ on each copy of $N$, is outward on $\widetilde{L}^{+}$, and is well defined and smooth at $L^{-}$. The construction of $\widetilde{\mathscr{X}}$ will follow Pugh [8]. On $P^{-}$, use the product structure $L^{-} \times(-\varepsilon, \varepsilon)$ to write $\mathscr{X}$ as $\mathscr{X}_{h}+\mathscr{X}_{v}$, where $\mathscr{X}_{h}$ and $\mathscr{X}_{v}$ are the horizontal and vertical components of $\mathscr{Z}$. Approximate $\mathscr{Z}_{h}$ on $P^{-}$by a smooth vectorfield $\mathscr{Z}_{h}$ so that $\mathscr{Z}_{h} \equiv \mathscr{X}_{h}$ on $\partial_{N}\left(P^{-}\right)$and $\mathscr{Z}_{h}$ has only nondegenerate critical points in $L^{-}$. As $\mathscr{X}$ is transverse to $L^{+}, \mathscr{X}_{h}$ is nonzero on $L^{+} \cap L^{-}$. Form the vectorfield

$$
\mathscr{Z}= \begin{cases}\mathscr{X} & \text { on } N \backslash P^{-}, \\ \mathscr{Z}_{h}+\mathscr{X}_{v} & \text { on } P^{-}\end{cases}
$$

For $x \in L^{-}$, use the $\mathscr{P}$-flow to put coordinates on $P^{-}$: denote $x \circ v$ as $(x, v)$. Let $\mathscr{Z}_{h}^{\prime}(x, v)$ be the parallel translate of $\mathscr{Z}_{h}(x, 0)$ along the vertical line to $(x, v) \in P^{-}$, and let $\rho: \mathbf{R} \rightarrow[0,1]$ be a $C^{\infty}$ bump function which is one on $[-\varepsilon / 2, \varepsilon / 2]$ and zero outside $[-\varepsilon, \varepsilon]$. Finally, define

$\widetilde{\mathscr{X}}=\left\{\begin{array}{lr}\mathscr{X}(x), & x \in \widetilde{N} \backslash \widetilde{P}^{-}, \\ \rho(v)\left[\mathscr{Z}_{h}^{\prime}(x, v)+|v| \mathscr{X}_{v}(x, v)\right]+(1-\rho(v)) \mathscr{Z}(x, v), & {[(x, v)] \in \widetilde{P}^{-} .}\end{array}\right.$

$\widetilde{\mathscr{X}}$ is now a well-defined $C^{1}$ vectorfield on $\tilde{N}$ which is outward on $\tilde{L}^{+}$. 
Note that the vertical component of $\widetilde{\mathscr{Z}}$ on $\widetilde{P}^{-}$is a positive multiple $\mathscr{Z}_{v}(x, v)$ for $v>0$, and is zero for $v=0$. As $\mathscr{Z}$ is transverse to $L^{-}, \mathscr{Z}_{v}$ is nonzero on a neighborhood of $L^{-}$, which may be taken to include $P^{-}$. That is, $\widetilde{\mathscr{X}}$ is nonzero on $\widetilde{P}^{-} \backslash L^{-}$, and all zeros lie in $\widetilde{S} \cup L^{-}$. Then by the Poincaré-Hopf theorem, $\chi(\widetilde{N})=\sum_{x \in \tilde{S} \cup L^{-}} l_{x}(\widetilde{\mathscr{X}})$.

Likewise, $\widetilde{\mathscr{X}} \mid L^{-}$is tangent to $L^{-}$and outward on $\partial L^{-}=L^{-} \cap L^{+}$, so $\chi\left(L^{-}\right)=\sum_{x \in L^{-}} l_{x}\left(\widetilde{\mathscr{X}} \mid L^{-}\right)$. But since $L^{-}$is the entrance set of $N, \mathscr{Z}_{v}$ points inward on $L^{-}$and $L^{-}$is a repelling set in the $\widetilde{\mathscr{X}}$ flow. That is, at every zero of $\widetilde{\mathscr{X}} \mid L^{-}, l(\widetilde{\mathscr{X}})=1$ and $l(\widetilde{\mathscr{X}})=\imath\left(\widetilde{\mathscr{X}} \mid L^{-}\right)$. Thus $\chi(\tilde{N})-\chi\left(L^{-}\right)=\sum_{x \in \tilde{S}} l(\widetilde{\mathscr{X}})$. But $\chi(\tilde{N})=2 \chi(N)-\chi\left(L^{-}\right)$, so

$$
2\left(\chi(N)-\chi\left(L^{-}\right)\right)=\sum_{x \in \widetilde{S}} l_{x}(\mathscr{X})=2 \sum_{x \in S} l_{x}(\mathscr{X}) .
$$

If $\mathscr{X}$ is replaced by $-\mathscr{X}$, then the roles of $L^{-}$and $L^{+}$are reversed, and

$$
\chi(M ; S)=\chi(N)-\chi\left(L^{+}\right)=\sum_{x \in S} l_{x}(-\mathscr{X})=(-1)^{n} \sum_{x \in S} l_{x}(\mathscr{X}) .
$$

All of the results for intersection numbers and the Hopf index (duality, stability, additivity, computability, etc.) can now be applied to the Conley-Euler numbers.

Corollary. For any isolated invariant set $S$ of a $C^{1}$ flow on a manifold $M$ :

(i) The forward and reverse indices are dual: $\chi^{+}(M ; S)=(-1)^{n} \chi^{-}(M ; S)$;

(ii) (Additivity) If $\left\{S_{p}\right\}$ is a collection of isolated invariant subsets of $S$ with all critical points in $S$ contained in $\bigcup S_{p}$, then $\chi(M ; S)=\sum \chi\left(M ; S_{p}\right)$;

(iii) (Products) If $S_{1}$ is isolated in $M_{1}$ and $S_{2}$ is isolated in $M_{2}$, then $\chi\left(M_{1} \times M_{2} ; S_{1} \times S_{2}\right)=\chi\left(M_{1} ; S_{1}\right) \chi\left(M_{2} ; S_{2}\right)$;

(iv) (Continuation) If $R\left(S_{\lambda}, \lambda\right)$ and $R\left(S_{\mu}, \mu\right)$ are related by continuation in $\mathscr{C}(\Lambda)$, then $\chi\left(M_{\lambda} ; S_{\lambda}\right)=\chi\left(M_{\mu} ; S_{\mu}\right)$;

(v) If $\chi(M ; S)$ is nonzero, then there exists a rest point of the flow in $S$;

(vi) There exist arbitrarily small perturbations of $\mathscr{X}$ such that $S$ continues to an isolated invariant set $S^{\prime}$ containing at least $|\chi(M ; S)|$ critical points, with critical points hyperbolic.

At most of the results can be derived in restricted settings from corresponding properties of the homology Conley index, some comments on the relation of this corollary to previous results are in order.

(i) As was noted above, duality for the Conley-Euler numbers follows from duality for the homology index when $M$ is orientable. Duality for the homology index fails if no neighborhood of $S$ in $M$ is orientable.

(ii) If the sets $S_{p}$ form a Morse decomposition of $S$, then additivity follows from the Morse inequalities [3]. The additivity of the Conley-Euler numbers gives less information than the Morse inequalities, but holds in much greater 
generality. Consider, for example, the circle with $( \pm 1,0)$ as rest points, flow counter-clockwise elsewhere. This flow has $S_{1}=(1,0)$ and $S_{2}=(-1,0)$ as isolated invariant sets, with homology Conley indices,

$$
\begin{aligned}
& C H_{*}\left(S^{1} ; S^{1}\right)=-(\mathbf{Z}, \mathbf{Z}, 0, \ldots), \\
& C H_{*}\left(S^{1} ; S_{1}\right)=(0,0,0, \ldots), \\
& C H_{*}\left(S^{1} ; S_{2}\right)=(0,0,0, \ldots) .
\end{aligned}
$$

These satisfy the Euler number equation, but not the Morse inequalities.

(iii) This also follows from the product formula for homology index.

(iv) The Conley-Euler number is much more stable than the Conley index itself. Take the family of flows on $\mathbf{R}^{2}$ generated by vectorfields

$$
\left\{\begin{array}{l}
\dot{r}=r(\lambda-r), \\
\dot{\varphi}=1 .
\end{array}\right.
$$

For $\lambda \neq 0$, the sets $S_{1 \lambda}=\{r=0\}$ and $S_{2 \lambda}=\{r \leq \lambda\}$ are isolated invariant sets, with indices $\Sigma^{2}$ and $\sum^{0}$ respectively. $S_{10}=S_{20}$ is isolated with index $\Sigma^{0}$. $S_{2 \lambda}$ is related by continuation to $S_{20}$ if $\mathscr{S}(\Lambda)$, but $S_{1 \lambda}$ is not. However, both have $R\left(S_{1 \lambda}, \lambda\right)$ related by continuation to $R\left(S_{20}, 0\right)$ in $\mathscr{C}(\Lambda)$, and $\chi\left(S_{1 \lambda}\right)=$ $\chi\left(S_{2 \lambda}\right)$.

(v) The same result is obtained in [5] as a corollary of the Lefschetz theorem for the Conley index, without the smoothness assumptions.

(vi) Again, when the critical points form a Morse decomposition (such as a gradient flow), the lower bound follows from the Morse inequalities. Of course, even in the classical setting of a compact manifold, it may not be possible to achieve the lower bound of exactly $|\chi(M ; S)|$ critical points.

If $M$ is a compact oriented manifold without boundary, then $M$ is isolated in itself, and $\chi(M)=(-1)^{n} \chi(M ; M)=I(\mathscr{Z})$. Similarly, if $S$ is a repeller in $M$, then there is an index triple $\left(N, \varnothing, L^{+}\right)$for $S$ in $M$. That is, $N$ can be chosen to be a manifold with boundary, with $\mathscr{Z}$ outward on $\partial N=L^{+}$. Then $\chi(N)=(-1)^{n} \chi(M ; S)=I(R(S))=I(\mathscr{Z} \mid N)$. Thus the theorem contains as special cases the classical absolute and relative versions of the Poincare-Hopf theorem.

Another special case and application of the theorem are developed in [1]. To show that a class of equations modelling competition of species exhibit exclusion (i.e. have no attractor corresponding to all species surviving), Armstrong and McGehee first show that there are not attracting critical points. To do so, they employ attracting blocks for attractors, and define the Euler number of an attractor $A$ to be the Euler number of an attracting block. In the language of this paper, an attracting block is an index triple of the form $(N, \partial N, \phi)$, and the Euler number of the block is the Conley-Euler number of the attractor. An attracting critical point would then have Euler number \pm 1 . However, the class of equations they consider can be perturbed to be fixed-point free, so a Hopf index theorem argument shows that any attractor must have an Euler number of zero. 


\section{REFERENCES}

1. R. Armstrong and R. McGehee, Some mathematical problems concerning the ecological problem of competitive exclusion, J. Differential Equations 23 (1977), 30-52.

2. C. Conley, Isolated invariant sets and the Morse index, CBMS Regional Conf. Ser. in Math., no. 38, Amer. Math. Soc., Providence, R.I., 1978.

3. C. Conley and E. Zehnder, Morse-type index theory for flows and periodic solutions for Hamiltonian equations, Comm Pure Appl. Math. 37 (1984), 207-253.

4. M. Hirsch, Differential topology, Springer-Verlag, Berlin, 1976.

5. C. McCord, Mappings and homological properties in the Conley index theory, Ergodic Theory Dynamical Systems 8 (1988), 175-198.

6. __ Poincaré-Lefschetz duality for the homology Conley index, preprint.

7. J. Milnor, Topology from the differentiable viewpoint, Univ. Press of Virginia, Charlottesville, 1965.

8. C. Pugh, A generalized Poincaré index formula, Topology 7 (1968), 217-226.

9. J. Robbin and D. Salamon, Dynamical systems, shape theory and the Conley index, Ergodic Theory Dynamical Systems 8 (1988), 375-394.

10. D. Salamon, Connected simple systems and the Conley index of isolated invariant sets, Trans. Amer. Math. Soc. 291 (1985), 1-41.

Department of Mathematics, University of Cincinnati, Cincinnati, Ohio 45221 